\title{
Interstitial Ti for intermediate band formation in Ti-supersaturated silicon
}

\author{
D. Pastor, ${ }^{1,2, a)}$ J. Olea, ${ }^{2}$ A. Muñoz-Martín, ${ }^{3}$ A. Climent-Font, ${ }^{3,4}$ I. Mártil, ${ }^{1}$ \\ and G. González-Díaz ${ }^{1}$ \\ ${ }^{1}$ Departamento de Física Aplicada III (Electricidad y Electrónica), Facultad de Ciencias Físicas, \\ Universidad Complutense de Madrid, Ciudad Universitaria s/n, 28040 Madrid, Spain \\ ${ }^{2}$ Instituto de Energía Solar, Escuela Técnica Superior de Ingenieros de Telecomunicación, \\ Universidad Politécnica de Madrid, Ciudad Universitaria s/n, 28040 Madrid, Spain \\ ${ }^{3}$ CMAM, Universidad Autónoma de Madrid, Campus de Cantoblanco, E-28049 Madrid, Spain \\ ${ }^{4}$ Departamento de Física Aplicada, Universidad Autónoma de Madrid, Campus de Cantoblanco, \\ E-28049 Madrid, Spain
}

(Received 30 October 2012; accepted 1 November 2012; published online 7 December 2012)

\begin{abstract}
We have analyzed by means of Rutherford backscattering spectrometry (RBS) the Ti lattice location and the degree of crystalline lattice recovery in heavily $\mathrm{Ti}$ implanted silicon layers subsequently pulsed laser melted (PLM). Theoretical studies have predicted that Ti should occupy interstitial sites in silicon for a metallic-intermediate band (IB) formation. The analysis of Ti lattice location after PLM processes is a crucial point to evaluate the IB formation that can be clarifyied by means of RBS measurements. After PLM, time-of-flight secondary ion mass spectrometry measurements show that the Ti concentration in the layers is well above the theoretical limit for IB formation. RBS measurements have shown a significant improvement of the lattice quality at the highest PLM energy density studied. The RBS channeling spectra reveals clearly that after PLM processes Ti impurities are mostly occupying interstitial lattice sites. (C) 2012 American Institute of Physics. [http://dx.doi.org/10.1063/1.4768274]
\end{abstract}

\section{INTRODUCTION}

In the last few years, much effort has been focused on improving the photovoltaic conversion efficiency of solar cells. In the framework of the third generation of solar cells, the intermediate band (IB) idea has emerged as a promising candidate to overcome the efficiency of the conventional Shockley-Queisser thermodynamical limit of single junction solar cells. ${ }^{1,2}$ The introduction of an IB inside the band gap of a semiconductor would allow the absorption of sub-band gap photons. These low energy photons could promote electrons from the valence band to the conduction band via the IB, contributing with additional electrons to enhance the solar cell efficiency. In recent years, several experimental investigations have demonstrated IB formation in highly mismatched alloys (HMA) and quantum dots. ${ }^{3,4}$ Another suggested way to obtain an IB is the introduction of deep level impurities into the host semiconductor at concentrations that exceed the metallic Mott limit $\left(\sim 5.9 \times 10^{19} \mathrm{~cm}^{-3}\right) .^{5}$

Silicon-based research in the IB materials field has attracted great interest due to the potential to increase the efficiency of the silicon solar cells, which comprise the $90 \%$ of the solar cell market. Supersaturated silicon with calchogenides $^{6}$ or Ti (Ref. 7) are being investigated extensively. These IB materials have been fabricated using the combination of techniques that are out of thermodynamical equilibrium; such as the ion implantation followed by pulsed laser melting (PLM). With these techniques, we obtain silicon with the required $\mathrm{Ti}$ impurity concentration to exceed the Mott limit while retaining a high crystal quality. In order to evaluate the PLM effects on the crystallinity of the Ti

${ }^{\text {a)} E l e c t r o n i c ~ m a i l: ~ d p a s t o r @ ~ f i s . u c m . e s . ~}$ implanted Si layers, an exhaustive analysis has been done in this work.

Theoretical predictions, based on the density functional theory (DFT), have suggested that the formation of an IB in $\mathrm{Ti}$ supersaturated silicon could take place only when $\mathrm{Ti}$ is located in an interstitial site. ${ }^{8}$ These theoretical calculations predict that $\mathrm{Ti}$ impurities occupying substitutional lattice positions would form an empty IB. This behavior is not desirable since a certain carrier population is required in the IB to pump electrons from the IB to the conduction band independently of the carrier charge concentration pumped from the valence band to the IB. ${ }^{5}$ Recent UV-Raman studies of the heavily Ti-implanted Si layers have suggested Ti interstitial presence associated to a new observed vibrational mode at $600 \mathrm{~cm}^{-1}$. 9

We have recently reported electrical transport properties of $\mathrm{Ti}$ supersaturated $\mathrm{Si}$ fabricated with the same conditions that exhibit an anomalous electrical decoupling behavior at low temperatures. This electrical behavior has been explained in terms of IB formation. ${ }^{10,11}$ In addition, strong sub-band gap absorption has been observed in Ti-supersaturated $\mathrm{Si}$ samples. This absorption is not related to defects or free carrier absorption and has been attributed to the IB formation. ${ }^{12}$

In this paper, we investigate the $\mathrm{Ti}$ lattice location in $\mathrm{Ti}$ supersaturated Si layers by means of channeling Rutherford backscattering spectrometry (RBS) measurements. Ti concentration profile obtained by means of time-of-flight secondary ion mass spectrometry (ToF-SIMS) measurements show that after PLM a Ti concentration above the theoretical Mott limit is obtained. We also find a high lattice recovery by means RBS measurements after the PLM performed at $0.8 \mathrm{~J} / \mathrm{cm}^{2}$. 


\section{EXPERIMENTAL}

Single crystal n-type silicons (thickness $=300 \mu \mathrm{m}$; $\mu=1450 \mathrm{~cm}^{2} / \mathrm{Vs} ; \mathrm{n}=2.2 \times 10^{13} \mathrm{~cm}^{-3}$, (111) grown direction) were implanted in a VARIAN CF3000 Ion Implanter at $33 \mathrm{keV}$ with $\mathrm{Ti}$ at doses: $10^{15}, 5 \times 10^{15}, 10^{16}$, and 5 $\times 10^{16} \mathrm{~cm}^{-2}$ using a $7^{\circ}$ tilt angle to minimize ion channeling effects. After implantation, samples were PLM processed with a $\mathrm{KrF}$ excimer laser $(248 \mathrm{~nm})$ at 0.2 and $0.8 \mathrm{~J} / \mathrm{cm}^{2}$ with a single pulse of $20 \mathrm{~ns}$ at J. P. Sercel Associates Inc. (New Hampshire, USA). Ti implantations were simulated with Montecarlo numerical calculations program SRIM. ${ }^{13}$

Depth profiles of Ti-implanted Si were obtained by ToFSIMS characterizations. These measurements were carried out with a TOF_SIMS IV model manufactured by ion-TOF, using a $25 \mathrm{keV}$ pulsed $\mathrm{Bi}^{3+}$ beam at $45^{\circ}$ incidence. Details of ToF-SIMS measurements can be found elsewhere. ${ }^{11}$

RBS measurements were carried out at the 5 MV Cockroft-Walton tandetron accelerator of the Centro de MicroAnálisis de Materiales (CMAM) in the Universidad Autónoma de Madrid. The vacuum of the chamber was $5 \times 10^{-6} \mathrm{~Pa}$. For the measurements, $2 \mathrm{MeV} \mathrm{He}{ }^{+}$ions were used. The scattered ions were detected simultaneously by two $\mathrm{Si}$ semiconductor particle detectors, placed at scattering angles of $126^{\circ}$ and $171^{\circ}$. The measurements were performed orientating the samples at random and channeling geometry in the direction (111). Numerical simulations of the RBS spectra were obtained using a standard backscattering simulation package (SIMNRA). ${ }^{14}$

\section{RESULTS}

In Fig. 1(a), we show the ToF-SIMS depth profiles of Ti-implanted Si samples with doses of $10^{15}$ and $10^{16} \mathrm{~cm}^{-2}$ before and after PLM process at $0.8 \mathrm{~J} / \mathrm{cm}^{2}$. SRIM profile simulations for these implantation doses are also showed. The $\mathrm{Ti}$ profiles of all the as-implanted samples present the typical gaussian-like profile and are well above the theoretical limit for the IB formation. For the sample implanted with a

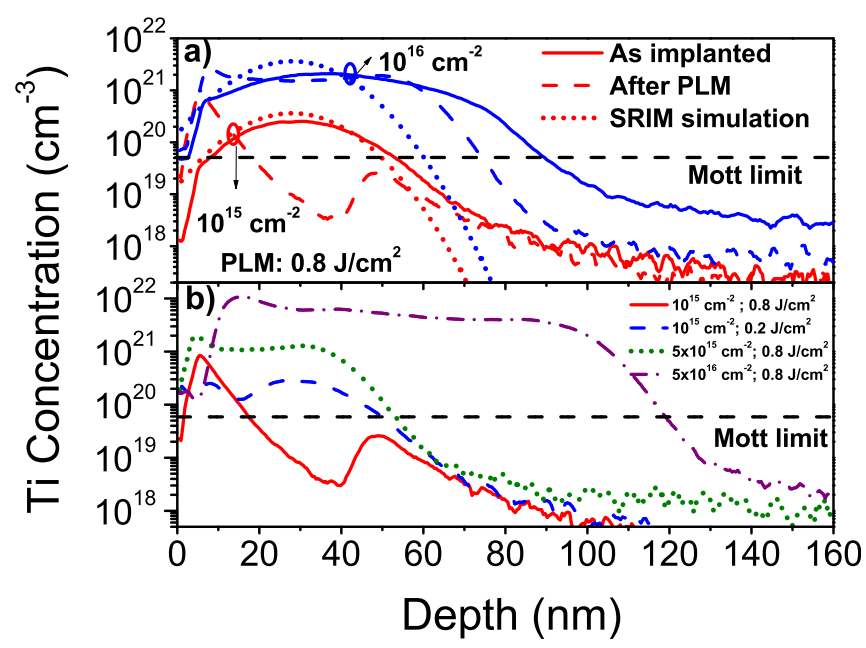

FIG. 1. (a) ToF-SIMS profiles of Ti-implanted Si samples with $10^{15}$ and $10^{16} \mathrm{~cm}^{-2}$ before and after PLM processes at $0.8 \mathrm{~J} / \mathrm{cm}^{2}$. SRIM profiles simulation of as-implanted samples at $10^{15}$ and $10^{16} \mathrm{~cm}^{-2}$ are also shown. (b) ToF-SIMS profiles of Ti-implanted Si samples with $10^{15}$ at 0.2 and $0.8 \mathrm{~J} / \mathrm{cm}^{2}, 5 \times 10^{15} \mathrm{~cm}^{-2}$ at $0.8 \mathrm{~J} / \mathrm{cm}^{2}$, and $5 \times 10^{16} \mathrm{~cm}^{-2}$ at $0.8 \mathrm{~J} / \mathrm{cm}^{2}$.
$10^{15} \mathrm{~cm}^{-2}$ dose, the SRIM simulations of as-implanted samples is in good agreement with as-implanted Ti depth profile of the ToF-SIMS measurements, except in the deeper region where the Ti depth profiles exhibit a long end tail that has been produced by the channeling residual effects of the ion implantation. Conversely, for the sample implanted with the highest dose, there are higher differences between SRIM simulations and ToF-SIMS measurements. This could be because of this implanted dose presents a most significantly channeling effect that cannot be simulated with the SRIM code.

After the PLM processes performed at $0.8 \mathrm{~J} / \mathrm{cm}^{2}$, the Ti depth profiles still have a high Ti concentration well above the Mott limit. This confirms that thermal processes performed in non equilibrium permit to obtain the impurity concentration required to IB formation when the solubility limit is exceeded. ${ }^{15}$ This PLM process has been extensively used after ion implantation to obtain IB materials in the highly mismatched alloys and supersaturated silicon. ${ }^{6,16}$ It is worth noting that these non thermodynamical equilibrium techniques have been employed recently to achieve a good lattice quality in deep level impurity supersaturated Si layers. ${ }^{17}$

To form an IB material, it is essential to obtain a Ti concentration above Mott limit. In the ToF-SIMS profiles of Figs. 1(a) and 1(b), a clear Ti impurity push effect toward the surface can be observed for all the analyzed samples. ${ }^{17}$ This push effect is produced during the PLM process when a superficial layer of the sample is melted, and the impurities tend to homogeneously spread in the melted region, because the diffusion coefficients and the solubility limit are orders of magnitude higher than in the solid phase. In the solidifying stage, the melted front moves toward the surface of the $\mathrm{Si}: \mathrm{Ti}^{+}$layer. Since the solubility limit in the solid phase is in orders of magnitude lower than in the melted phase, a push effect takes place, displacing the impurities toward the surface of the sample. This push effect is clearly visible for the lowest implantation dose $\left(10^{15} \mathrm{~cm}^{2}\right)$ where a narrow and superficial $\mathrm{Ti}$ concentration peak is observed. Although this push effect is not so evident in the figure for the sample implanted with a dose one order of magnitude higher $\left(10^{16} \mathrm{~cm}^{-2}\right)$, Ti concentration profile redistribution can be clearly appreciated. The two different implanted doses present a strong contrast when SIMS profiles before and after the PLM annealing are compared. Whereas, in the sample implanted with a $10^{15} \mathrm{~cm}^{-2}$ dose, the strong push effect reduces substantially the depth with a Ti concentration above the Mott limit, in the sample doped with $10^{16} \mathrm{~cm}^{-2}$, this reduction is not so accentuated.

Figure 1(b) displays ToF-SIMS profiles of samples with representative doses and PLM energy densities that we are going to discuss for RBS measurements. In this figure, it can be appreciated that all the samples after PLM for the different implanted doses preserve a Ti impurity concentration well above the Mott limit. We estimate the width of the implanted layer from ToF-SIMS profiles by considering the width for which the Ti concentration is $90 \%$ of the total. These widths are 20,50, and $90 \mathrm{~nm}$ for samples implanted with $10^{15}, 5 \times 10^{15}$, and $5 \times 10^{16} \mathrm{~cm}^{-2}$ doses and PLM processed at $0.8 \mathrm{~J} / \mathrm{cm}^{2}$, respectively. Comparing the Ti profiles of 


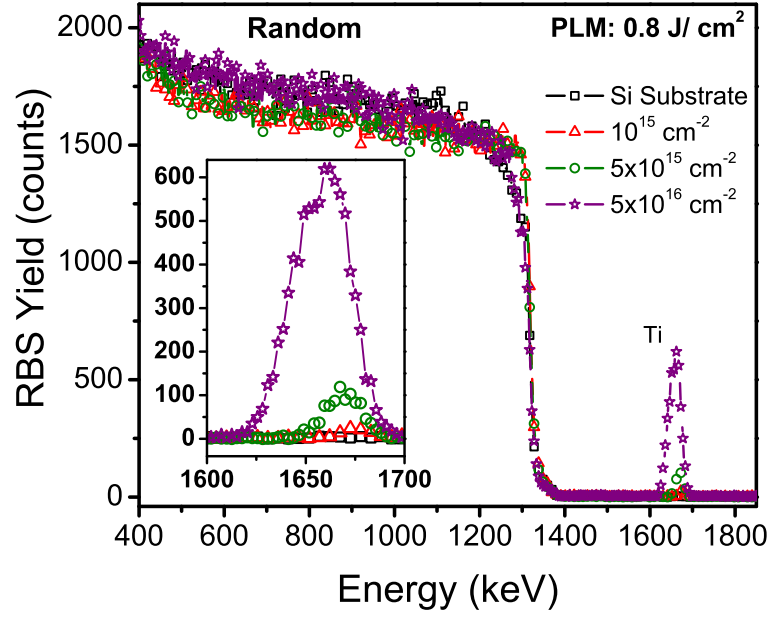

FIG. 2. RBS spectra in random configuration of Ti-implanted Si samples at $10^{15}, 5 \times 10^{15}$, and $5 \times 10^{16} \mathrm{~cm}^{-2}$ doses and PLM processed at $0.8 \mathrm{~J} / \mathrm{cm}^{2}$. RBS spectrum of the Si reference substrate is also shown.

the samples implanted with a dose of $10^{15} \mathrm{~cm}^{-2}$ and PLM processed at 0.2 and $0.8 \mathrm{~J} / \mathrm{cm}^{2}$ energy densities with the asimplanted sample with the same dose (see Fig. 1(a)), we can observe that the $\mathrm{Ti}$ profile at the lowest energy density annealing exhibits only a slight push effect. For this sample, the Ti superficial peak is present but with a visible reduced concentration, suggesting a lower $\mathrm{Ti}$ loss than the sample PLM processed at the highest energy density.

Fig. 2 shows the RBS spectra of Ti-implanted Si samples at $10^{15}, 5 \times 10^{15}$, and $5 \times 10^{16} \mathrm{~cm}^{-2}$ doses and subsequently PLM processed at $0.8 \mathrm{~J} / \mathrm{cm}^{2}$. The RBS spectrum of the unimplanted silicon substrate is also shown for comparative purposes. All these spectra were acquired in random configuration. As it can be observed, the low energy side of the spectra, up to about $1400 \mathrm{keV}$, shows the contribution of the Si substrate with similar shape for all the samples. At higher energies, between 1600 and $1700 \mathrm{keV}$, the signals from the implanted Ti showed different widths and heights depending on the implanted dose. As implanted dose is increased, this peak exhibits an increase in the intensity that is related to the higher Ti concentration introduced in the lattice by ion implantation. The inset of Fig. 2 shows in detail the energy region of the Ti peak. The area below of the different curves of this energy is proportional to the Ti concentration. A progressive broadening can be observed for the $\mathrm{Ti}$ related peak as implanted dose is increased, reaching a significant broadening for the sample with the highest implanted dose. In this case, where the Ti incorporation has been performed by ion implantation, this peak broadening reflects the Ti incorporation in a deeper region in agreement with the ToF-SIMS measurements showed in Fig. 1.

Figure 3(a) shows the RBS spectra of the sample implanted with the $10^{15} \mathrm{~cm}^{-2}$ doses and PLM processed at $0.8 \mathrm{~J} / \mathrm{cm}^{2}$ in random geometry together with the SIMNRA simulation. As it can be observed, the SIMNRA fit reproduces faithfully the RBS spectrum considering the limitations in the depth resolution of the measurements. To reach this approximation, we have introduced in the simulation a very thin superficial Si layer in the top of the Ti impurified Si layer to
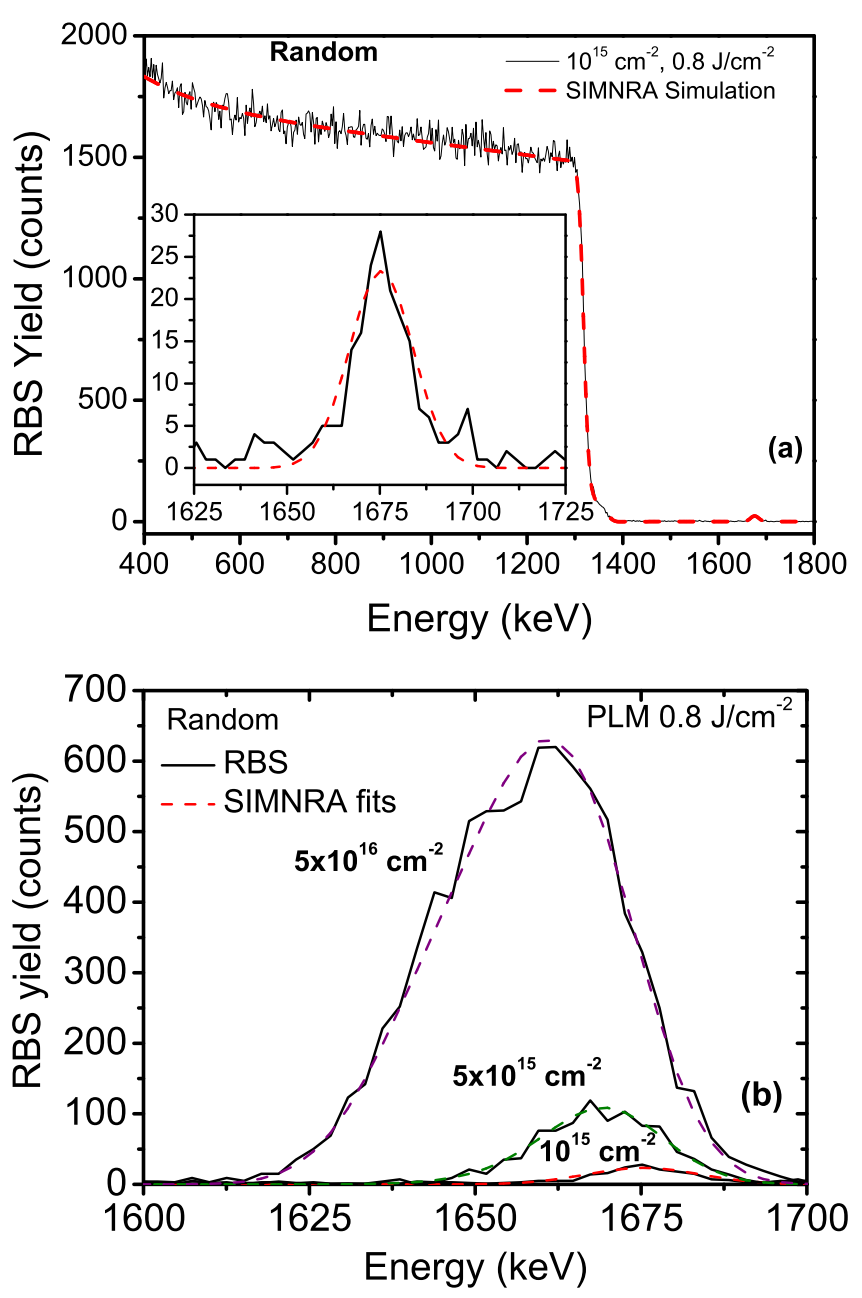

FIG. 3. (a) RBS spectra in random configuration and SIMNRA simulations of Ti-implanted Si samples at $10^{15} \mathrm{~cm}^{-2}$ dose and PLM processed at $0.8 \mathrm{~J} / \mathrm{cm}^{2}$. The inset displays details of the Ti related peak. (b) RBS spectra and SIMNRA simulations of Ti-implanted Si samples at $10^{15}, 5 \times 10^{15}$, and $5 \times 10^{16} \mathrm{~cm}^{-2}$ doses and PLM processed at $0.8 \mathrm{~J} / \mathrm{cm}^{2}$ centered in the Ti peak region.

take into account that $\mathrm{Ti}$ concentration begin to be significant from $5 \mathrm{~nm}$ below the surface. The inset displays the Ti related peak with both experimental and SIMNRA simulated spectra. Though there are slight differences between the SIMNRA fit and the experimental spectrum, it can be observed that SIMNRA simulation reproduces Ti peak energy location. The rest of random spectra for the different doses has been also simulated obtaining similar results. From these fittings, an average thickness of Si layer with Ti implanted can be estimated: $20 \mathrm{~nm}$ for the sample implanted with $10^{15} \mathrm{~cm}^{-2}, 45 \mathrm{~nm}$ for the sample implanted with $5 \times 10^{15} \mathrm{~cm}^{-2}$, and $180 \mathrm{~nm}$ for the sample implanted with $5 \times 10^{16} \mathrm{~cm}^{-2}$. These results are very similar to that obtained from ToF-SIMS measurements and there are some differences only for the highest implanted dose. The Ti doses of the samples after PLM process obtained from the SIMNRA fitting: $1.7 \times 10^{15}, 8.7 \times 10^{15}$, and $7.5 \times 10^{16} \mathrm{~cm}^{-2}$ are clearly overestimated with respect to the respective implanted doses of $10^{15}, 5 \times 10^{15}$, and $5 \times 10^{16} \mathrm{~cm}^{-2}$. This is because of the depth resolution of these RBS measurements $(40-50 \mathrm{~nm})$ which are lower in comparison with the ToF-SIMS measurements $(5-10 \mathrm{~nm})$. Additionally, the RBS measurements in the case of $\mathrm{Ti}$ 
detection present a statistical noise quite accentuated due to the low experimental intensity signal obtained. The statistical noise $\sqrt{N}$ is directly related to the counts number (N).

Figure 3(b) shows the RBS spectra and SIMNRA simulations of Ti-implanted $\mathrm{Si}$ samples at $10^{15}, 5 \times 10^{15}$, and $5 \times 10^{16} \mathrm{~cm}^{-2}$ doses and PLM processed at $0.8 \mathrm{~J} / \mathrm{cm}^{2}$. As it has been commented previously, differences between the RBS measurements and the SIMNRA fit simulations are due to limited depth resolution and the statistical noise. In the case of the RBS spectra represented in this figure, the statistical noise $(\sqrt{N})$ can be expressed as $N \pm \sqrt{N}$, resulting in $30 \pm 5$, $100 \pm 10$, and $620 \pm 25$ for the $10^{15}, 5 \times 10^{15}$, and $5 \times 10^{16} \mathrm{~cm}^{-2}$ Ti-implanted $\mathrm{Si}$ samples, respectively; i.e., corresponds to an error of the $16 \%, 10 \%$, and $4 \%$.

To analyze the crystal quality of the Ti-supersaturated samples, in Fig. 4, we show the RBS spectra of implanted samples at $10^{15} \mathrm{~cm}^{-2}$ processed with a PLM energy density of 0.2 and $0.8 \mathrm{~J} / \mathrm{cm}^{2}$ in random and channeling geometry. As it can be observed, whereas the peak related with $\mathrm{Ti}$ is still present in the channeling spectra, a peak related to dechanneling effects in the superficial layer, emerges around $1300 \mathrm{keV}$. This peak reflects the lack of $\mathrm{Si}$ lattice quality because of $\mathrm{Si}$ atoms displaced of their lattice positions during Ti ion implantation. In the case of Si substrate, the presence of very low intensity peak is due to dechanneling effects on the surface. These spectra have not been simulated due to that SIMNRA does not reproduce channeling spectra. As it can be seen, the sample PLM processed at the lowest energy density displays a peak in the channeling spectrum with a high intensity with respect to $\mathrm{Si}$ substrate, indicating a poor lattice quality since only a small quantity of $\mathrm{Si}$ atoms returns to their lattice positions. In contrast, the spectrum of the sample PLM processed at $0.8 \mathrm{~J} / \mathrm{cm}^{2}$ shows a peak around $1300 \mathrm{keV}$ with a very low intensity, clearly similar to the silicon substrate, pointing out to a very high lattice reconstruction after ion $\mathrm{Ti}$ implantation and PLM process and consequently a high lattice quality. These results are clearly in agreement with glancing incidence $\mathrm{x}$-ray measurements, ${ }^{18}$ TEM images, electron diffaction (ED) (Ref. 17) analysis and Raman scattering measurements ${ }^{9}$ have been reported previously, demonstrating that the highest energy density used is enough to recover the Si crystal lattice.

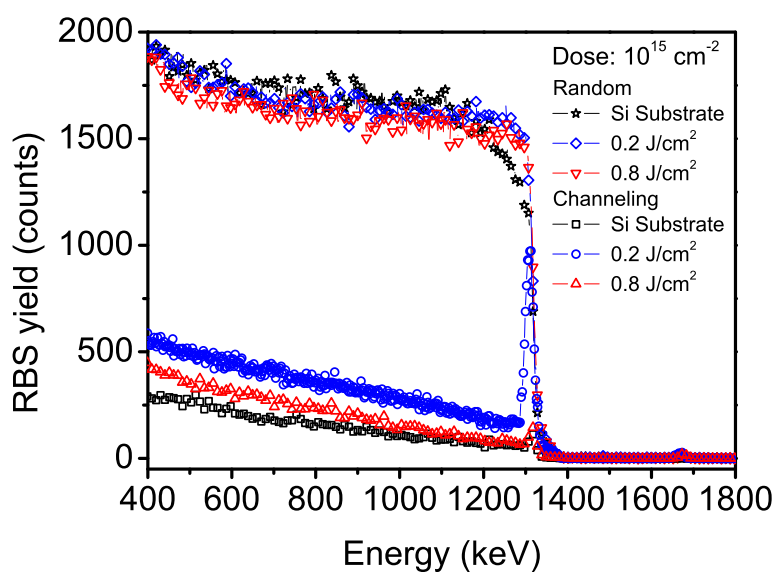

FIG. 4. RBS spectra of Ti-implanted Si samples at $10^{15} \mathrm{~cm}^{-2}$ and PLM processed at 0.2 and $0.8 \mathrm{~J} / \mathrm{cm}^{2}$ oriented in random and channeling geometry. Si substrate RBS spectrum is also shown.

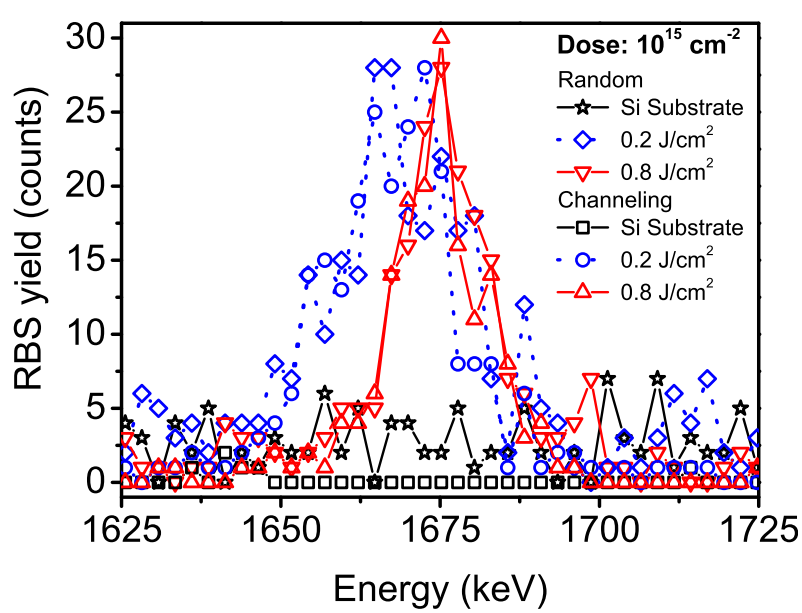

FIG. 5. RBS spectra centered at the Ti peak signal for the $\mathrm{Si}$ samples implanted with $10^{15} \mathrm{~cm}^{-2}$ and PLM processed at 0.2 and $0.8 \mathrm{~J} / \mathrm{cm}^{2}$ at random and channeling configuration. RBS spectra of the Si substrate sample are also shown.

Finally to analyze the lattice position of the Ti atom, we display in Fig. 5 the Ti related peak for the Ti implanted samples with $10^{15} \mathrm{~cm}^{-2}$ and PLM processed at 0.2 and $0.8 \mathrm{~J} / \mathrm{cm}^{2}$. For the sample implanted with a dose of $10^{15} \mathrm{~cm}^{-2}$ and PLM processed at $0.8 \mathrm{~J} / \mathrm{cm}^{2}$, there are no appreciable differences in the intensity between the spectra in channeling or random configuration, indicating that $\mathrm{Ti}$ atoms are massively occupying interstitial lattice positions in the host semiconductor. From the slight differences observed in the area of the curves in all the samples implanted with different doses (not shown here), we can conclude that the percentage of Ti interstitial is over $95 \%$. On the other hand, theoretical calculations reported recently ${ }^{8}$ predict a metallic IB formation inside the Si lattice only when the Ti atoms are located at certain interstitials positions, but not in substitutional positions.

Additionally, in Fig. 5, it can be observed also the differences in the Ti related peak in the spectra of the samples PLM processed at the lowest and highest energy density $(0.2$ and $0.8 \mathrm{~J} / \mathrm{cm}^{2}$ ). These spectra are acquired in the both configurations, channeled and random. The spectra of the samples PLM processed at $0.8 \mathrm{~J} / \mathrm{cm}^{2}$ show narrower peaks and a peak displacement towards higher energies (which means towards the surface of the implanted layer), in contrast with the spectra of the samples PLM processed at the low energy $\left(0.2 \mathrm{~J} / \mathrm{cm}^{2}\right)$. The FWHM of the Ti related peaks in the RBS spectra are $20 \mathrm{keV}$ and $35 \mathrm{keV}$, for the 0.8 and $0.2 \mathrm{~J} / \mathrm{cm}^{2}$ energy densities, respectively. All these point out that the $\mathrm{Ti}$ impurities in the samples PLM processed at $0.8 \mathrm{~J} / \mathrm{cm}^{2}$ are concentrated in a reduced superficial depth. This indicates also that high density energy during the PLM process produces diffusion of the impurities to the surface layer in completely agreement with the ToF-SIMS results presented previously.

\section{CONCLUSIONS}

We show that samples implanted at very high concentrations preserve after PLM processes, Ti concentrations well above the theoretical Mott limit. We have demonstrated that Ti depth concentration obtained from SIMS and RBS 
measurements are in clear agreement, and show that a very good crystal lattice can be achieved for the lowest implanted dose at the maximum energy density investigated. We find in the analyzed samples a massive interstitial position of $\mathrm{Ti}$ impurities after PLM processed. The electronic transport properties in these samples have been satisfactorily explained previously in terms of the IB formation in the $\mathrm{Ti}$ supersaturated Si layer. This result could point out, contrary to the classical understanding of doping, that a substitutional lattice position could not be essential to obtain an electrical activation in the case of an IB formation in agreement with previous theoretical calculations.

\section{ACKNOWLEDGMENTS}

Authors would like to acknowledge the C. A. I. de Técnicas Físicas of the Universidad Complutense de Madrid for ion implantation experiments, Nanotechnology and Surface Analysis Services of the Universidad de Vigo C.A.C.T.I. for ToF-SIMS measurements, and C.M.A.M. of the Universidad Autónoma de Madrid for RBS measurements.

This work was partially supported by the Project NUMANCIA II (Grant No. S-2009/ENE/1477) funded by the Comunidad de Madrid. D. Pastor and J. Olea acknowledge Professor A. Luque and Professor A. Martí for the useful discussions and guidance and also acknowledge financial support from the MICINN within the program Juan de la Cierva (JCI-2011-10402 and JCI-2011-11471).
${ }^{1}$ A. Luque and A. Martí, Phys. Rev. Lett. 78, 5014 (1997).

${ }^{2}$ W. Shockley and H. J. Queisser, J. Appl. Phys. 32, 510 (1961).

${ }^{3}$ N. López, A. Reichertz, K. M. Yu, K. Campman, and W. Waluckiewicz, Phys. Rev. Lett. 106, 028701 (2011).

${ }^{4}$ A. Luque, A. Martí, and C. Stanley, Nat. Photonics 6, 146 (2012).

${ }^{5}$ A. Luque, A. Martí, E. Antolín, and C. Tablero, Physica B 382, 320 (2006).

${ }^{6}$ B. P. Bob, A. Kohno, S. Charnvanichborikarn, J. M. Warrender, I. Umezu, M. Tabbal, J. S. Williams, and M. J. Aziz, J. Appl. Phys. 107, 123506 (2010).

${ }^{7}$ G. Gonzalez-Díaz, J. Olea, I. Mártil, D. Pastor, A. Martí, E. Antolín, and A. Luque, Sol. Energy Mater. Sol. Cells 93, 1668 (2009).

${ }^{8}$ K. Sánchez, I. Aguilera, P. Palacios, and P. Wahnón, Phys. Rev. B 79, 165203 (2010).

${ }^{9}$ D. Pastor, J. Olea, A. del Prado, E. García-Hemme, I. Mártil, G. GonzálezDíaz, J. Ibáñez, R. Cuscó, and L. Artús, Semicond. Sci. Technol. 26, 115003 (2011).

${ }^{10}$ J. Olea, G. González-Díaz, D. Pastor, I. Mártil, A. Martí, E. Antolín, and A. Luque, J. Appl. Phys. 109, 063718 (2011).

${ }^{11}$ D. Pastor, J. Olea, A. del Prado, E. García-Hemme, R. García-Hernansanz, and G. González-Díaz, Sol. Energy Mater. Sol. Cells 104, 159-164 (2012).

${ }^{12}$ J. Olea, A. del Prado, D. Pastor, I. Mártil, and G. González-Díaz, J. Appl. Phys. 109, 113541 (2011).

${ }^{13}$ J. F. Ziegler et al., SRIM-The Stopping and Range of Ions in Matter (SRIM, 2011).

${ }^{14}$ M. Mayer, SIMNRA User's Guide, Report IPP 9/113, Max-Planck-Institut fur Plasmaphysik, Garching, Germany, 1997.

${ }^{15}$ S. Hoccine and D. Mathiot, Appl. Phys. Lett. 53, 1269 (1988).

${ }^{16}$ K. M. Yu, W. Waluckiewickz, J. Wu, W. Shan, J. W. Beeman, M. A. Scarpulla, O. D. Dubon, and P. Becla, Phys. Rev. Lett. 91, 246403 (2003).

${ }^{17}$ J. Olea, M. Toledano-Luque, D. Pastor, E. San-Andrés, I. Mártil, and G. González-Díaz, J. Appl. Phys. 107, 103524 (2010).

${ }^{18}$ J. Olea, M. Toledano-Luque, D. Pastor, G. González-Díaz, and I. Mártil, J. Appl. Phys. 104, 016105 (2008). 